\title{
Tabularia
}

\section{Redocumentariser la Tapisserie de Bayeux : base de données documentaire et système d'informations spatialisées}

Redocumentarising the Bayeux Tapestry: Document database and spatialised information system

Ridocumentare l'Arazzo di Bayeux : base dati documentaria e sistema

d'informazioni spazializzate

Antoine Verney, Clémentine Berthelot, Arnaud Daret, Jérôme Chauveau, Pierre-Yves Buard, Julia Roger et Cécile Binet

\section{OpenEdition}

\section{Journals}

Édition électronique

URL : http://journals.openedition.org/tabularia/3278

DOI : 10.4000/tabularia.3278

ISSN : $1630-7364$

Éditeur :

CRAHAM - Centre Michel de Boüard, Presses universitaires de Caen

\section{Référence électronique}

Antoine Verney, Clémentine Berthelot, Arnaud Daret, Jérôme Chauveau, Pierre-Yves Buard, Julia Roger et Cécile Binet, "Redocumentariser la Tapisserie de Bayeux : base de données documentaire et système d'informations spatialisées », Tabularia [En ligne], Les sources des mondes normands à I'heure du numérique, mis en ligne le 19 décembre 2018, consulté le 20 avril 2019. URL : http:// journals.openedition.org/tabularia/3278 ; DOI : 10.4000/tabularia.3278 



\title{
Redocumentariser la Tapisserie de Bayeux: base de données documentaire et système d'informations spatialisées
}

\author{
Redocumentarising the Bayeux Tapestry: \\ Document database \\ and spatialised information system \\ Ridocumentare l'Arazzo di Bayeux: \\ base dati documentaria \\ e sistema d'informazioni spazializzate
}

$\begin{array}{ll}\begin{array}{l}\text { Antoine VERNEY } \\ \text { Musée de Bayeux }\end{array} & \begin{array}{l}\text { Clémentine BERTHELOT } \\ \text { Musée de Bayeux } \\ \text { Arnaud DARET } \\ \text { Certic, Unicaen }\end{array} \\ \text { Jérôme CHAUVEAU } \\ \text { Certic, Unicaen } \\ \begin{array}{l}\text { Pôle Document numérique, } \\ \text { MRSH, Unicaen }\end{array} & \text { Julia RoGER } \\ \end{array}$

Cécile BINET

Drac Normandie

Résumé:

Dans le cadre du projet de refonte du Musée de la Tapisserie de Bayeux à l'horizon 2024, mené par la Ville de Bayeux et la DRAC Normandie, en collaboration avec la région Normandie et le département Calvados, des outils numériques permettant de découvrir et d'étudier la Tapisserie sous un angle nouveau ont été élaborés.

Ces outils consistent en un «Système d'information documentaire spatialisée» (SIDS), combinaison d'un système documentaire et d'un système d'information spatialisé. Ils offrent des solutions de gestion et de valorisation des ressources documentaires relatives à la Tapisserie de Bayeux, et doivent à terme accompagner l'ensemble des publics qui s'intéressent à l'œuvre, notamment les chercheurs en sciences humaines, les conservateurs-restaurateurs, les spécialistes du textile, le grand public. Ce projet est le fruit d'un étroit partenariat entre l'État, la Ville de Bayeux, l'université de Caen Normandie, le CNRS et l'Ensicaen.

Mots-clés: Tapisserie de Bayeux, campagne de numérisation, traitement d'image, information spatialisée, base documentaire, structuration de données, standards internationaux (XML, TEI, EAD), extraction de données documentaires

Tabularia "Études", «Les sources des mondes normands...", 2018, p. 1-27, 19 décembre 2018 URL: http://journals.openedition.org/tabularia/3278 | DOI: 10.4000/tabularia.3278 
Abstract:

Alongside the renewal of the Bayeux Tapestry Museum planned for 2024, led by the city of Bayeux, the DRAC Normandy, the Normandy region and the Calvados department, several numeric tools have been created to discover and examine the Tapestry in new ways.

Those tools consist in a Spatialised Document Information System (SDIS), combining a Document database and a Spatialised information system. They offer solutions to manage and to enhance documentary resources related to the Bayeux Tapestry and should, at the end, accompany all the persons interested in the Tapestry, particularly scholars in Humanities, curators-restorers, specialists in textile and the public. This project is the result of a close collaboration between the State, the city of Bayeux, the University of Caen Normandy, the CNRS and the Ensicaen.

Keywords: Bayeux Tapestry, digitization campaign, image processing, spatialized information, document database, data structuring, international standards (XML, TEI, EAD), extraction of documentary data

Riassunto:

Nell'ambito del progetto di ristrutturazione del Museo dell'Arazzo di Bayeux che dovrebbe concludersi nel 2024, portato avanti dalla Città di Bayeux e dalla DRAC Normandie, in collaborazione con la regione Normandia e il dipartimento del Calvados, sono stati elaborati nuovi strumenti informatici che permettono di scoprire e studiare l'Arazzo da un nuovo punto di vista.

Questi strumenti consistono in un «Sistema d'informazione documentaria spazializzata (SIDS), combinazione di un sistema documentario e di un sistema d'informazione spazializzato. Essi offrono delle soluzioni di gestione e di valorizzazione delle fonti documentarie relative all'A razzo di Bayeux, e devono accompagnare i vari fruitori dell'opera, soprattutto i ricercatori in scienze umane, i conservatori-restauratori, gli specialisti del tessile, il grande pubblico. Questo progetto è il frutto di uno stretto partenariato tra lo Stato, la Città di Bayeux, l'Università di Caen Normandie, il CNRS e l'ENSICAEN.

Parole chiave: Arazzo di Bayeux, campagna di informatizzazione, trattamento d'immagini, informazione spazializzata, base documentaria, strutturazione di dati, standards internazionali (XML, TEI, EAD), estrazione di dati documentari

\section{Introduction}

La «Tapisserie de Bayeux» est une broderie de laine sur toile de lin du $\mathrm{XI}^{\mathrm{e}}$ siècle retraçant l'histoire de l'accession au trône d'Angleterre par Guillaume le Conquérant. Il s'agit d'une œuvre d'art monumentale, exceptionnelle par sa taille - près de $70 \mathrm{~m}$ de long sur $70 \mathrm{~cm}$ de large - ainsi que par le témoignage qu'elle nous rapporte sur la vie au $\mathrm{XI}^{\mathrm{e}}$ siècle. Elle est classée au titre des Monuments historiques en 1840, et au registre «Mémoire du Monde» de l'UNESCO en 2007. Il s'agit d'une propriété de l'État, déposée au sein de la collection muséographique de la Ville de Bayeux «Tapisserie de Bayeux-Musée d'Art et d'Histoire Baron Gérard »1.

1. Collection bénéficiant de l'appellation Musée de France, JO du 29/07/2017. 
La Tapisserie de Bayeux est considérée comme un chef-d'œuvre de l'art roman et une source unique pour la compréhension de la création du royaume anglo-normand. Elle est ainsi un sujet d'étude extrêmement discuté, analysé, documenté, malgré le manque de ressources apportant des informations sur son origine. En effet, la première source documentaire reconnue abordant cette œuvre date de $1476^{2}$. Par conséquent, les questions relatives à la naissance de l'œuvre restent ouvertes, encore à ce jour, malgré la densité des études sur le sujet.

Ainsi, la connaissance de cette œuvre est nourrie constamment depuis que la communauté scientifique s'est réapproprié l'œuvre il y a près de trois siècles, dès sa "redécouverte» par le monde savant par le biais de deux académiciens, Antoine Lancelot et dom Bernard de Montfaucon, donnant lieu à une bibliographie extrêmement riche et variée. Le poids de cette bibliographie illustre toute la diversité des disciplines qui ont tenté d'offrir un regard particulier sur cette œuvre: histoire, histoire de l'art, art plastique, archéologie, linguistique, littérature, etc. à travers un grand nombre de nationalités représentées parmi les chercheurs, démontrant un rayonnement international de cette œuvre majeure du Moyen Âge ${ }^{3}$. Shirley-Ann Brown a tenté de faire la synthèse de la bibliographie relative à la Tapisserie de Bayeux: le résultat de son ambitieux travail rend compte de la masse des contributions à son étude, notamment des deux côtés de la Manche ${ }^{4}$.

Parallèlement, les études relatives à l'analyse scientifique de la Tapisserie de Bayeux en tant qu'objet textile sont moins nombreuses et plus récentes ${ }^{5}$. Parmi elles, l'ambitieuse campagne d'études qui s'est déroulée de novembre 1982 à janvier 1983, lors du transfert de l'œuvre de son ancienne vitrine de l'Hôtel du Doyen à l'actuelle au Centre Guillaume le Conquérant, a donné lieu à un lot documentaire important: relevés inédits effectués sur le revers, prélèvements, tableaux d'analyse, rapports, clichés de détails de l'endroit et du revers. À ce jour, ces données n'ont fait l'objet d'aucune interprétation au-delà des rapports d'études eux-mêmes réalisés par les différentes équipes ayant participé à cette campagne, et il reste de nombreuses questions en suspens. De plus, une campagne d'imagerie multi-spectrale a été réalisée sur l'œuvre en janvier 2017. Ces études et données sont indispensables pour dresser le constat d'état matériel complet de l'œuvre et doivent être analysées de manière transversale, notamment en prenant appui sur les nouvelles technologies; celles-ci donnant la possibilité aujourd'hui de mettre en place des solutions d'exposition des données documentaires facilitant leur manipulation.

2. Inventaire du trésor de la Cathédrale de Bayeux.

3. Voir, par exemple, parmi les contributions les plus récentes: PAGÈs I PARETAS, 2015; Henderson et Owen-Crocker (éd.), 2016; Lemagnen, Brown et Owen-Crocker, à paraître.

4. BROWN, 2013.

5. Voir, par exemple, les différents bilans de la campagne d'étude de 1982-1983 dans La Tapisserie de Bayeux..., 2004, p. 67-123 et des analyses de 2015-2017 dans Lemagnen, Brown et OwenCrocker, à paraitre. 
La gestion documentaire est donc un volet particulièrement riche pour la valorisation de la Tapisserie, par sa densité, mais aussi par la diversité des disciplines et donc des vocabulaires utilisés pour son étude, dans de multiples langues. L'accès à cette masse d'information est une problématique prédominante pour la compréhension de l'œuvre dans toutes les dimensions que son étude permet de mettre en valeur: en tant qu'œuvre d'art, document sur la vie au $\mathrm{XI}^{e}$ siècle et récit d'un évènement majeur pour l'Histoire européenne.

Les enjeux importants posés par l'accès à l'œuvre, la densité de la bibliographie $^{6}$, la dispersion de certaines ressources documentaires uniques, la nature même de la Tapisserie - très longue -, l'absence d'un système de référence consensuel pour se repérer dans l'œuvre... sont autant de problématiques pour lesquelles l'outil numérique peut offrir des solutions. Il s'agit là de l'un des objectifs majeurs du projet «Musée de la Tapisserie de Bayeux/Centre de Compréhension de l'Europe du Moyen Âge», projet de refonte du Musée de la Tapisserie de Bayeux à l'horizon 2024, mené par la Ville de Bayeux en collaboration étroite avec l'État (Drac Normandie), la région Normandie et le département Calvados.

Ce projet est né en 2013, de la volonté commune de l'État, propriétaire de l'œuvre, et de la Ville de Bayeux, dépositaire, d'améliorer les conditions de conservation, de présentation et d'accès à la Tapisserie de Bayeux. Parmi les études qui président à l'effectivité de ce projet, le volet numérique est particulièrement ambitieux, puisqu'il doit offrir des solutions de gestion et de valorisation des ressources documentaires relatives à l'œuvre, et accompagner à différents niveaux l'ensemble des types de publics qui s'intéressent à la Tapisserie: chercheurs en sciences humaines, conservateurs / restaurateurs spécialistes du textile, grand public... Ce volet numérique se matérialise par un projet de Système d'information documentaire spatialisée (qu'on appellera SIDS ${ }^{7}$ ), dont la création est le fruit d'un étroit partenariat conventionné entre l'État, la ville de Bayeux, l'université de Caen Normandie et le CNRS, à travers trois laboratoires coordonnés par la direction de la Maison de la recherche en sciences humaines (MRSH): le Certic (DSI, Unicaen), le Greyc (Unicaen / Ensicaen /CNRS) et le Pôle Document Numérique (MRSH, CNRS / Unicaen). Son enrichissement, envisagé sur le long terme, met en jeu la collaboration de nombreuses institutions gestionnaires de fonds documentaires, notamment l'État et ses établissements publics et services à compétence nationale, le conseil

6. Dans la publication des actes du colloque de Cerisy-la-Salle en 1999, La Tapisserie de Bayeux, L'art de broder l'Histoire, François Neveux, en fait le constat dans son introduction : «Les publications sur la Tapisserie sont, en effet, si nombreuses qu'il est difficile aux chercheurs de s'y retrouver», Neveux, 2004, p. 11.

7. SIDS est la contraction de SID pour Système d'information documentaire (ensemble organisé de ressources qui permet de regrouper, de classifier, traiter et diffuser l'information sur un environnement donné) et SIS pour Système d'information spatialisée (Système informatique permettant de rassembler, d'organiser, de combiner, d'analyser et de présenter des informations localisées spatialement, contribuant à la compréhension d'un espace qui dans notre cas est la Tapisserie elle-même). 
départemental et ses services, le conseil régional et les établissements publics à vocation régionale, spécialisés dans le patrimoine culturel.

Dans le présent article, nous présenterons d'abord le système documentaire puis le système d'information spatialisé élaborés dans le cadre du projet SIDS Tapisserie de Bayeux en montrant en quoi l'interaction de ces deux outils constitue pour les spécialistes un moyen d'étudier la Tapisserie sous un angle nouveau.

\section{Système documentaire}

L'objectif visé par le système documentaire est la redocumentarisation ${ }^{8}$ de la Tapisserie qui tient notamment compte de l'hétérogénéité des ressources. Cette contrainte, issue de la nature des documents indexés, implique la conception d'un système à plusieurs niveaux assurant la description des données au sein d'un univers commun. Citons, à titre d'exemples, quelques-uns des traits de cette hétérogénéité:

- hétérogénéité géographique: les documents sont dispersés dans différents lieux et conservés selon des modalités variées (fonds d'archives, institutions, fonds privés);

- hétérogénéité typologique: les documents sont formellement hétérogènes (texte, image ${ }^{9}$, composite ${ }^{10}$, sur support papier ou numérique) et scientifiquement hétérogènes (article, rapport, etc.).

$\mathrm{Au}$ sein du système documentaire ici présenté, il convient de distinguer au premier niveau les ressources documentaires préexistantes relatives à l'état matériel de l'œuvre rassemblées en interne (infra, 1.1.2), au deuxième niveau, les ressources textuelles préexistantes ou générées par le système documentaire (infra, 1.2), puis au troisième niveau, les ressources distantes à indexer au système documentaire (infra, 1.3).

8. La notion de redocumentarisation est un concept issu des sciences de l'information théorisé par le groupe de recherches collectif Roger T. Pédauque. Il désigne le second traitement documentaire que connaissent les documents à l'ère numérique. Il renvoie plus largement au passage d'un type de documentation à un autre: le système documentaire issu de l'imprimé s'appuie sur une intrication de la description de l'information conjointe fond / forme (texte/support) tandis que les technologies numériques impliquent la distinction des deux. Les conditions de production et d'échanges de documents numériques, passant par l'enrichissement des métadonnées, modifient la manière dont l'information se trouve identifiée et localisée. Voir notamment PÉDAUQUE, SALAÜN et MELOT, 2006 et SALAÜN, 2007

9. Jusqu'à un certain point, cette hétérogénéité se subdivise elle-même: par exemple, les documents iconographiques sont déclinables en différentes sous-catégories: il peut s'agir de gravures, de dessins au trait, de photographies se subdivisant elles-mêmes en prises de vues multi-spectrales, photomontage, etc. Il s'agit évidemment de rendre compte de ces particularités dans la description des données sans porter préjudice à la clarté de l'information pour le lecteur.

10. Un document pouvant contenir un contenu varié: images, tableaux, dessins, gravures, graphiques, etc. 


\subsection{Base documentaire}

\subsubsection{Contexte}

La nécessité de concevoir un système documentaire autour de la Tapisserie de Bayeux a été réaffirmée à l'occasion du lancement du projet de futur musée, en 2013. En effet, outre le besoin de rassembler en un seul point d'entrée la masse des travaux accumulée en trois siècles d'études, le projet de restructuration du musée s'articule autour de la volonté d'optimiser la valorisation de l'œuvre, tant au niveau de sa conservation/ restauration (constat d'état, restauration éventuelle), que de sa présentation au public (rendre à l'œuvre sa monumentalité), et du parcours muséographique accompagnant son interprétation. Tous ces points nécessitent une compréhension de l'œuvre toujours plus complète, de son état matériel à son iconographie.

Ainsi, la création de ce système documentaire s'attache à positionner la Tapisserie de Bayeux comme objet d'étude, et a pour objectif de réunir en un seul point un maximum de données documentaires passées (rapports d'étude, articles, relevés graphiques et photographiques, prélèvements, correspondances...) et futures, afin d'offrir un outil de référence au monde scientifique. Cet outil, à vocation collective et internationale, est également destiné à favoriser les liens entre les experts de toute discipline et nationalité, notamment français et britanniques. À terme, son interface sera proposée en français et en anglais.

\subsubsection{Présentation succincte des ressources documentaires et du plan de classement}

La base de données documentaire se compose de notices catalographiques qui pointent vers les documents référencés. Conçues comme les éléments d’un fonds d'archives ${ }^{11}$, ces notices sont structurées en $\mathrm{EAD}^{12}$ et réparties dans des collections selon un plan de classement que nous présentons ici succinctement.

Le plan de classement des fonds répertoriés s'articule autour des collections conservant des documents originaux ayant rapport à la Tapisserie. Celles-ci sont enrichies au fur et à mesure des récolements sur place, et selon deux objectifs majeurs:

- rassembler la documentation relative à l'étude de l'état matériel de l'œuvre, afin d'aider à la réalisation de l'étude préalable de la Tapisserie, c'est-à-dire un constat d'état avant étude de l'éventualité d'une campagne de stabilisation ou de restauration de l'œuvre. Ce travail sera lancé au cours du second

11. Cette interprétation est conforme à la proposition du collectif Roger T. Pédauque qui préconise de retourner aux racines des sciences de l'information, en particulier à l'archivistique, pour décrire des documents.

12. Encoding Archival Description: http://www.bnf.fr/fr/professionnels/formats_catalogage/a.f_ead. html. 
semestre 2018, ce qui en fait une priorité dans la conception de la base de données documentaire et une première étape;

- permettre aux experts d'autres disciplines de disposer d'un outil de visualisation et d'interprétation des données relatives à l'étude de l'iconographie de l'œuvre. Cette perspective, plus large et pour laquelle la documentation est bien plus dense, est étudiée en un second temps avec le comité scientifique du projet, composé d'historiens, d'historiens de l'art et d'archéologues.

Ainsi, la première étape à l'enrichissement de la base de données documentaire réside en l'indexation de la documentation relative à l'état matériel de l'œuvre. À ce jour, huit collections composent le plan de classement de la base, pour 2155 objets enregistrés: celles du Musée de la Tapisserie, de la DRAC Normandie, de l'Inventaire général du patrimoine culturel de Normandie, de la Médiathèque de l'architecture et du patrimoine, du Laboratoire de recherche des monuments historiques (LRMH), du Laboratoire interuniversitaire des systèmes atmosphériques (LISA), du Centre de recherche et de restauration des musées de France ( $\mathrm{C}_{2} \mathrm{RMF}$ ) et de la Fabrique de patrimoines en Normandie. Les objets indexés dans chacune de ces collections sont sélectionnés pour leur apport à la connaissance de l'œuvre au sens large, brassant un grand nombre d'objets d'études (étude des broderies, des faiblesses de la toile, du contexte de présentation de l'œuvre, etc.) afin de garantir à l'utilisateur l'accès à un maximum d'informations.

\section{Musée de la Tapisserie}

Le Musée abrite bien entendu un certain nombre de lots documentaires inédits permettant l'étude de l'état matériel de l'œuvre. Ont été indexées en priorité deux couvertures photographiques commandées par la Ville de Bayeux, en 1965 (lot «Joubert») et en 1983 (lot «La Goélette»). Les clichés photographiques ont l'avantage d'offrir un état de l'œuvre à un instant $t$; ces deux lots permettent de comparer deux états à presque deux décennies d'écart. Le contexte de présentation n'est pas le même entre ces deux séries, puisque la Tapisserie était exposée à l'Hôtel du Doyen entre 1948 et 1982, puis dans ses locaux actuels à partir de début 1983.

\section{DRAC Normandie}

Le siège de la Direction régionale des affaires culturelles, représentant de l'État, propriétaire de l'œuvre, conserve quelques ressources documentaires d'importance dans le suivi des différents projets de présentation qu'a connus la Tapisserie. Parmi ceux-ci, le relevé graphique établi par Naomi Moore, historienne de l'art, à l'occasion de la campagne d'étude de l'œuvre réalisée entre novembre 1982 et janvier 1983, nous offre une synthèse des évolutions de l'état de l'œuvre entre 1729 et 1871, grâce à son étude attentive de quatre sources documentaires de différentes époques: Foucault 1729; Stothard 1817-1819; Jubinal 1838; Dosseter 1871. 
Inventaire général du patrimoine culturel de Normandie (siège de Caen)

Cette institution qui dépend aujourd'hui de la région revêt une importance particulière puisque ses services conservent un lot conséquent de clichés photographiques réalisés lors de la campagne 1982-1983, notamment par Pascal Corbierre. Composé de 724 clichés indexés dans la base, ce lot offre une couverture photographique complète de l'œuvre dans sa vitrine de l'Hôtel du Doyen, et de multiples photographies de détails de l'endroit et, ce qui très important pour l'étude de l'œuvre, de son revers qui a été découvert uniquement à cette occasion.

Médiathèque de l'architecture et du patrimoine

La Médiathèque de l'architecture et du patrimoine conserve les archives de l'administration des Monuments Historiques. À ce titre, elle abrite un grand nombre de documentations sur la Tapisserie, premier objet mobilier classé monument historique sur la liste de 1840. Ainsi, différents lots provenant de ses collections ont été intégrés à la base de données du projet, et notamment les rapports d'études de la campagne 1982-1983 (rapports de synthèse sur les études réalisées sur le revers de l'œuvre ${ }^{13}$, sur la toile, dont une première analyse de la bande numérotée...), de nombreuses notes de travail (relevés des faiblesses de la toile ou des broderies au revers, inventaire des pièces de restauration, analyse de prélèvements...), ou des clichés parmi lesquels l'unique couverture photographique du revers de l'œuvre.

Laboratoire de recherche des monuments historiques (LRMH)

Ce laboratoire a notamment réalisé le suivi de l'état de l'œuvre depuis sa pose dans sa vitrine actuelle en 1983. Ainsi, quatre rapports décennaux ont été réalisés par le LRMH, en 1985, 1995, 2009 et 2014. L'objectif de ces rapports est de sélectionner puis de photographier tous les dix ans une cinquantaine de zones considérées comme fragiles (trous, taches, ruptures de fil, etc.), afin de comparer l'évolution de l'état matériel de l'œuvre.

Laboratoire interuniversitaire des systèmes atmosphériques (LISA)

Au cours des études opérées entre 2015 et 2017 à l'occasion du lancement du projet, le LISA a réalisé une étude sur l'état d'empoussièrement de la vitrine de la Tapisserie.

Centre de recherche et de restauration des musées de France ( $\left.\mathrm{C}_{2} \mathrm{RMF}\right)$

Les équipes d'imagerie du $\mathrm{C}_{2} \mathrm{RMF}$, à l'instar du LISA, ont participé aux études récentes sur l'œuvre dans son local actuel, en 2016. Ils ont opéré des tests d'imagerie sur une petite partie de l'œuvre (les trois premiers mètres) et l'étude de faisabilité de la campagne d'imagerie réalisée l'année suivante par la Fabrique

13. Bédat, Girault et Monier, 1982-1983. 
de patrimoines en Normandie. Leurs travaux ont donné lieu à une cinquantaine de clichés sous différentes lumières des toutes premières scènes.

La Fabrique de patrimoines en Normandie

Lors de la campagne de prises de vues multispectrales de la Fabrique de patrimoines en Normandie, en 2017, 1145 clichés ont été réalisés en lumière directe, réflexion infrarouge, fluorescence ultraviolet, réflexion ultraviolet, lumières rasantes basale et latérale. Cet ensemble constitue la campagne d'imagerie la plus complète réalisée sur la Tapisserie de Bayeux à ce jour.

L'ensemble des données indexées à partir de ces collections permettent, à différentes échelles, de renseigner l'état matériel de l'œuvre. C'est à ce titre qu'elles pourront être visualisées et interprétées par les utilisateurs de la base de données documentaire notamment au moment de la réalisation de l'étude préalable de l'œuvre. Ces collections ont vocation à être enrichies sur le long terme, parallèlement à d'autres collections qui resteront externes à cette base de données documentaire (cf. infra 1.3).

\subsection{Base de textes}

La base de textes réunira les travaux existants sur la Tapisserie elle-même (notamment les rapports et notes préparatoires de la campagne 1982-1983) ainsi que les travaux réalisés sur les aspects historiques et iconographiques à partir de la plateforme SIDS, qui a vocation à s'autoalimenter.

L'ensemble des textes intégrés à cette base sera structuré en XML TEI ${ }^{14}$ afin d'en permettre une intégration fluide dans le système documentaire. Les documents préexistants (rapports et notes préparatoires) seront numérisés, feront l'objet d'une reconnaissance optique des caractères, ou d'une transcription pour les documents manuscrits, puis d'une structuration en TEI. Un portail de consultation sera développé avec le moteur d'affichage $\mathrm{MaX}^{15}$ proposant une interrogation centralisée de l'ensemble des textes. Des expérimentations seront également menées, en collaboration avec des chercheurs du Greyc, pour annoter les renvois au système calque existants dans les textes (cf. infra) dans le but de permettre une interrogation depuis le module de visualisation de l'œuvre du SIDS.

\subsection{Indexation de ressources distantes}

Pour répondre au besoin de rassembler les travaux de l'ensemble de la communauté scientifique, d'autres collections de documents viendront également s'ajouter aux huit déjà indexées dans la base de données. Le but de ce système documentaire n'est pas de dupliquer certaines ressources documentaires existantes, mais d'indexer des ressources distantes à travers le système d'information documentaire

14. Text Encoding Initiative: http://www.tei-c.org/index.xml.

15. http://www.unicaen.fr/recherche/mrsh/document_numerique/outils/max. 
spatialisé. Ainsi, des chercheurs disposant d'une base d'informations scientifiques, quelle qu'en soit la nature - corpus de textes ou base catalographique - auront la possibilité d'indexer leurs données directement sur le système de visualisation en ligne de la Tapisserie, afin d'enrichir l'outil.

L'objectif est bien de proposer une solution d'indexation de référence permettant de localiser l'ensemble de l'information scientifique de manière homogène et, par là même, de fournir aux communautés concernées une solution d'accès aux données sur la Tapisserie la plus efficace possible.

\subsection{Choix technologiques et mise en ouvre}

Les technologies choisies pour intégrer les collections précédemment décrites dans la base de données sont les suivantes.

Le système documentaire est d'abord conçu comme un outil de travail permettant de mutualiser un ensemble d'objets hétérogènes et d'enrichir progressivement la connaissance de l'œuvre dans son aspect matériel, historique et iconographique. Le système doit garantir un accès unifié aux données, qu'il les agrège ou qu'il les produise, ainsi que la continuité et la pérennité du dialogue scientifique sur la Tapisserie tout au long du projet. D'autre part, comme on l'a vu plus haut, on considère l'ensemble documentaire comme un fonds d'archives dispersé dont la base de données est une reconstitution virtuelle permettant un accès unifié à la totalité des documents indexés. Cette double contrainte de l'accès à la fois unifié et pérenne aux documents fait naturellement écho à la problématique du SIDS qui doit garantir la possibilité de localiser et de visualiser de façon universelle et pérenne des informations sur la Tapisserie elle-même (voir infra, 2).

La conception des documents comme des archives (jusqu'à un certain point, on peut considérer la Tapisserie comme un méta-document qu'il s'agit de mettre en rapport avec les documents qui parlent d'elle) ainsi que la visée à long terme sont des critères qui président aux choix des techniques utilisées pour réaliser le système documentaire.

En considérant une approche orientée sur les données comme le cœur des dispositifs mis en place et non guidée par des choix logiciels, la base de données est développée dans le respect des standards internationaux de description des données et des normes en usage dans les domaines concernés par le projet (conservation/restauration, archives, patrimoine, histoire de l'art, histoire) à des fins d'échanges, d'interopérabilité et de pérennisation des ressources. Le format retenu pour les données est donc XML EAD. L'EAD est un format basé sur le langage XML qui permet de structurer des descriptions de manuscrits ou de documents d'archives.

La production de la base de données documentaire est divisée en deux tâches principales:

- l'enrichissement de la base en XML EAD natif, via un environnement de structuration ergonomique dédié. Cet outil et les aménagements d'interface 
permettent de saisir rapidement les informations et de voir les numérisations des documents concernés dès l'intégration des liens (fig. 1);

- la mise en place d'une solution d'exposition des données de la base documentaire. L'outil open source Pleade a été retenu pour remplir cette fonction, car sa mission première est de permettre la diffusion d'instruments de recherche archivistique dans une architecture web (fig. 2).

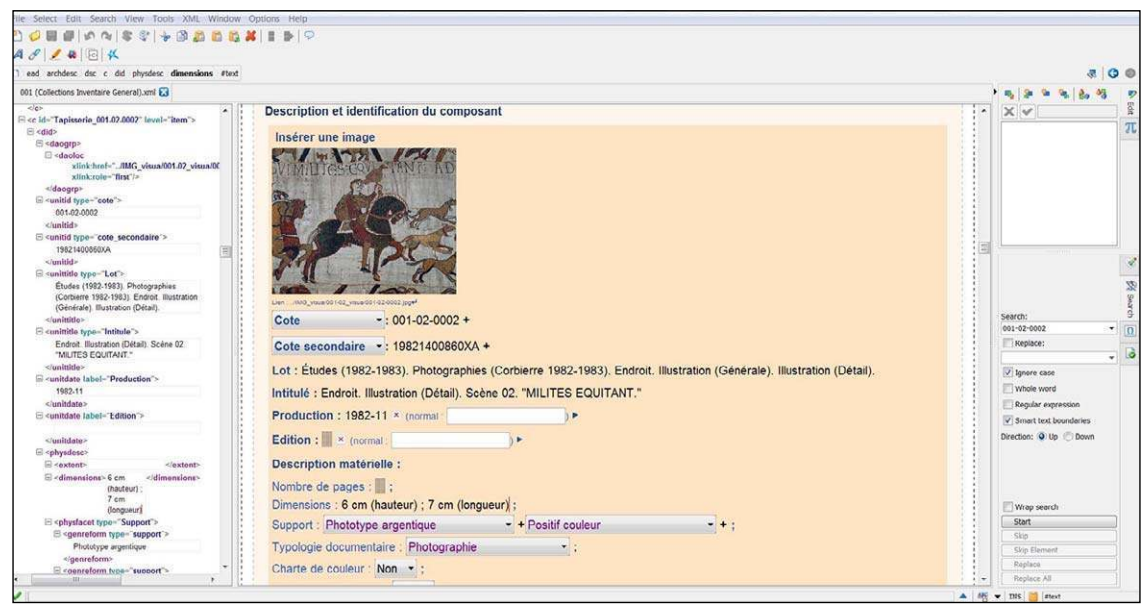

Fig. 1: Environnement de travail pour la structuration des données en XML EAD

Tapisserie de Bayeux

Collection de la Fabrique de patrimoines en Normandie

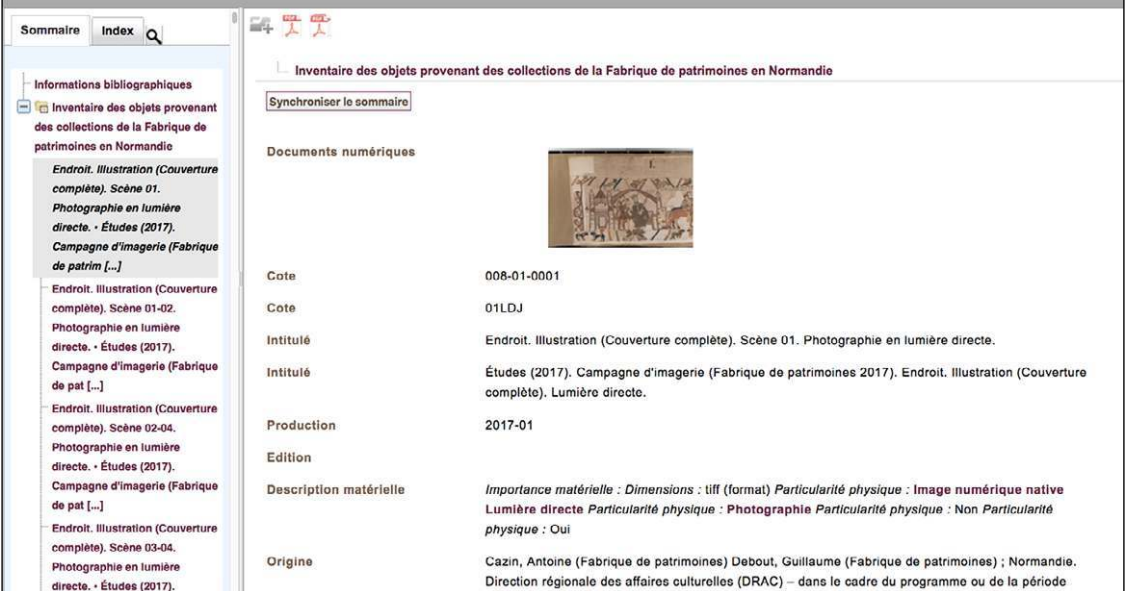

Fig. 2: Interface d'exposition des données de la base documentaire 


\section{Un système d'informations spatialisées}

Il existe une quantité très importante de données documentaires numériques ou numérisables sur la Tapisserie de Bayeux, autant sur le plan de l'étude de l'état matériel de l'œuvre (constats d'état, campagnes de relevés manuscrits et photographiques...), que sur des aspects purement historiques ou iconographiques. Si leur stockage ne pose pas de problème scientifique ou technique majeur - tant que l'on respecte bien l'utilisation de normes archivistiques connues et établies -, leur localisation dans l'espace d'étude - une broderie de près de $70 \mathrm{~m}$ de long sur $70 \mathrm{~cm}$ de large - ne fait pas l'objet d'un consensus et aucun mode de restitution à la fois accessible, dynamique, efficace et séduisant n'existe à ce jour.

En 1934, Hélène Chefneux dans son étude sur les fables dans la Tapisserie était catégorique à ce propos: "C'est à Bayeux même qu'il faut se rendre pour se faire une juste idée [...]; dans toutes les reproductions, elles sont réduites à une échelle si petite qu'elles deviennent presque inintelligibles. [...] De même, c'est de visu que doit être examinée la question des restaurations, parfaitement décelables à Bayeux ${ }^{16}$. Près d'un siècle plus tard et malgré l'explosion du numérique dans la vie quotidienne, ce constat est toujours de circonstance, et il demeure fortement compliqué de visualiser correctement et précisément cette œuvre autrement que par une visite au musée. Dans des études plus contemporaines, des présentations originales de l'œuvre ont été imaginées pour faciliter le suivi de la narration pour le lecteur, comme dans cette étude de Michel Parisse ${ }^{17}$ où la Tapisserie est représentée en couleur sur 3 lignes étalées sur 3 feuilles elles-mêmes pliées 3 fois (fig. 3). Ce procédé permet d'observer de larges parties de la Tapisserie sans pour autant être très précis, ni même pratique. Parmi les éditions numériques existantes de la Tapisserie, citons par exemple le travail de Martin Foys, universitaire américain spécialiste de littérature médiévale, qui a proposé en 2003 d'illustrer l'œuvre dans un environnement dédié, "a docuverse», où certaines sources majeures sont liées à la visualisation de l'œuvre elle-même en scrolling horizontal continu divisé en 173 panneaux ${ }^{18}$.

Partant du constat que représenter la Tapisserie de Bayeux d'un seul tenant sur les supports numériques actuels est impossible sans réduire la qualité de visualisation, l'idée a été d'adapter de manière originale les techniques utilisées de nos jours dans les SIS afin, d'une part, de fournir des modes de représentation avancées de l'œuvre avec une qualité suffisante pour être visualisée de manière globale ou précise, et, d'autre part, de permettre la superposition de couches d'informations thématiques et par la même occasion y indexer tout type de données qui s’y réfèrent.

16. Chefneux, 1934.

17. PARISSE, 1983.

18. FoYs, 2003 (http://www.sd-editions.com/bayeux/baytext2.jpg). 


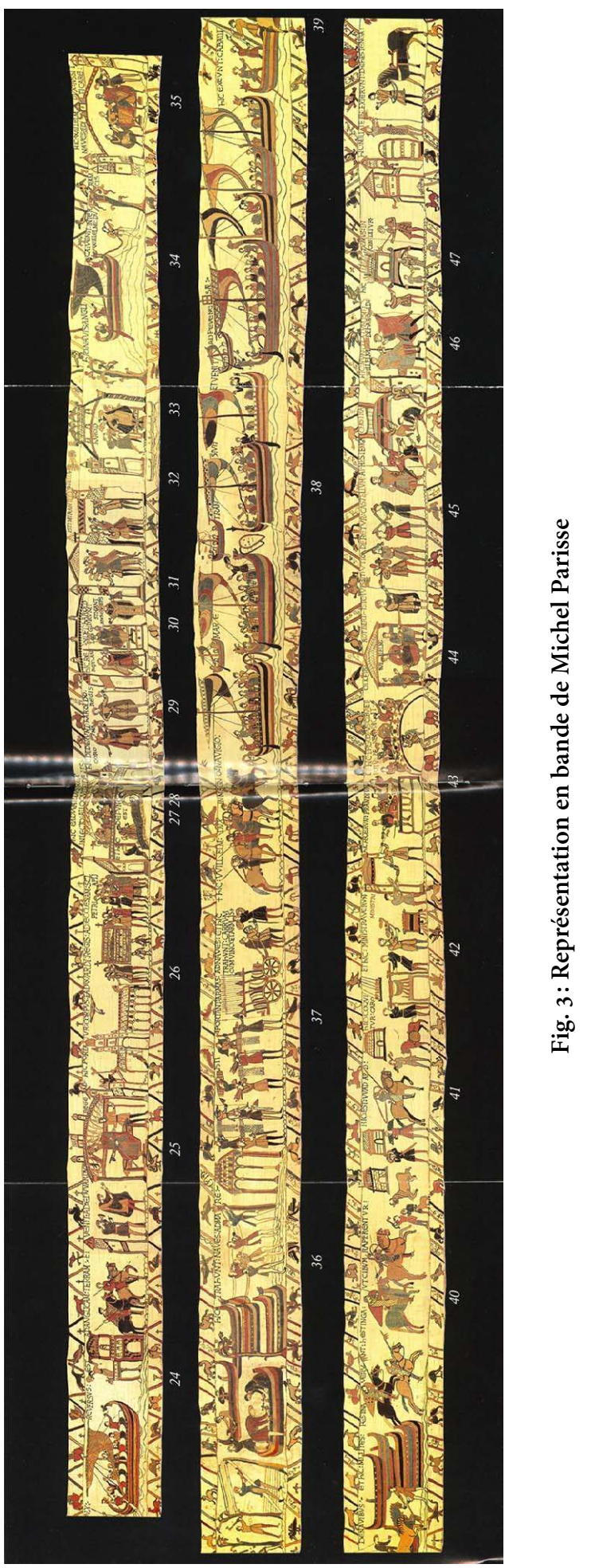

Tabularia «Études», «Les sources des mondes normands...», 2018, p. 1-27, 19 décembre 2018 


\subsection{La Tapisserie de Bayeux comme "fond de référence"}

Un SIS standard s'appuie sur un modèle d'informations spatialisées basé sur des couches qui se superposent pour caractériser et décrire un espace. Dans le cadre d'un $\mathrm{SIG}^{19}$, version géographique d'un système d'information spatialisé, cette superposition n'est possible que dans un même système de référence géographique $^{20}$ et en général la première de ces couches utilisée est une "carte» (ou «fond de carte»). Dans notre cas, l'espace d'étude est «fini», puisqu'il est délimité par les dimensions physiques de l'œuvre que nous devons donc être capables de représenter dans son intégralité. Cette représentation graphique complète de l'ouvre servira de "fond de carte» mais aussi de système de projection appliquée à la Tapisserie: c'est à travers cette réflexion que nous introduisons le concept de «fond de référence». Une reproduction de l'œuvre à partir de captures photo réalisées dans des conditions naturelles d'exposition ${ }^{21}$ servant à la fois d'espace d'étude mais aussi de repère dans un système de coordonnées cartésiennes. Une fois créé, ce fond servira à recaler d'autres fonds (prises de vues avec différentes modalités d'éclairage), couches thématiques ou toute donnée indexable permettant ainsi plus généralement le repérage, l'identification et le renseignement de portions de cet «espace fini» (voir fig. 4).

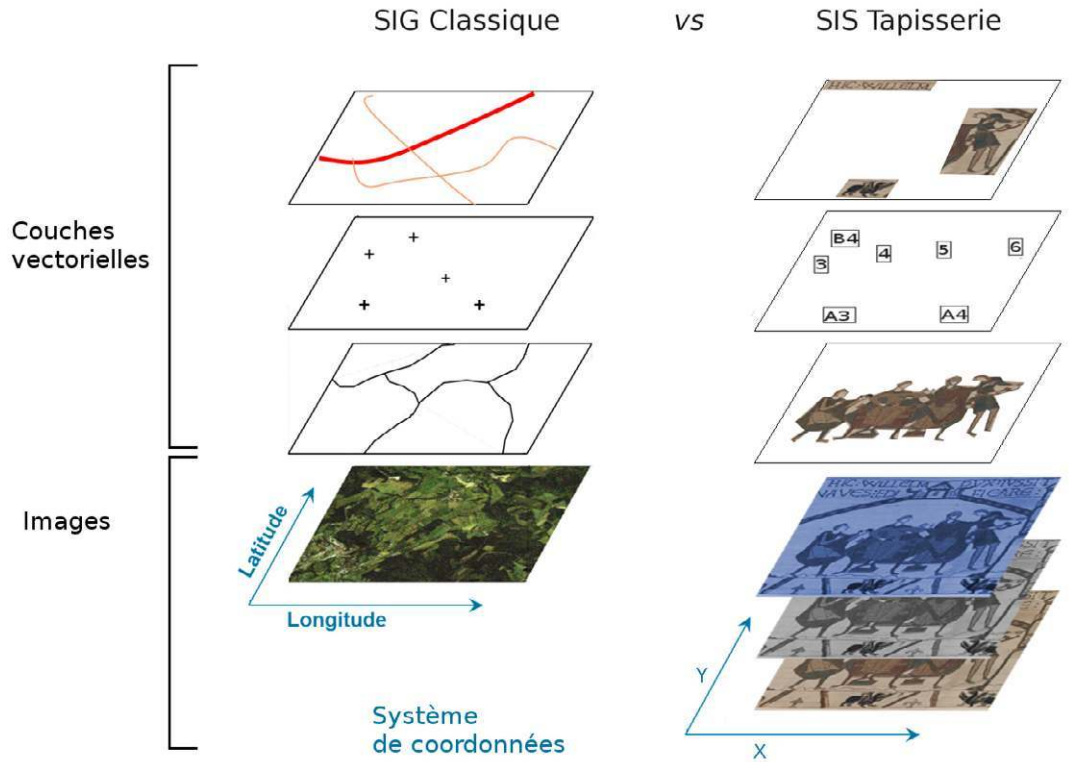

Fig. 4: Système d'information spatialisé de la Tapisserie de Bayeux

19. Système d'information géographique.

20. Nations-Unies, 2004, p. 226.

21. En lumière directe, encore appelée lumière du jour. Cette campagne a été réalisée par A. Cazin et G. Debout, de La Fabrique de patrimoines en Normandie, en janvier 2017. 
La première étape du travail a donc consisté à créer ce «fond de référence» qui doit représenter la Tapisserie le plus fidèlement possible. L'enjeu est important, même s'il est scientifiquement impossible de proposer une représentation unique de cette œuvre qui, par sa nature textile, est sujette de façon intrinsèque à des distorsions selon son mode d'accrochage ou les conditions hygrométriques dans lesquelles se trouve l'œuvre au moment de sa numérisation.

\subsubsection{Construction $d u$ fond de référence}

Depuis 1983, date de la dernière campagne de constat d'état, la Tapisserie est exposée au Centre Guillaume le Conquérant à Bayeux. L'ouvre y est placée dans un local technique séparé du déambulatoire où circulent les visiteurs par une vitre sécurisée, suspendue à un rail qui décrit un parcours en forme de parabole. Sans une extraction de l'œuvre de ces conditions de présentation, toute numérisation de la Tapisserie ne donnerait pas satisfaction ${ }^{22}$. Seul un accès direct à l'œuvre peut permettre une telle captation qui, dans tous les cas, ne peut être réalisée d'un seul tenant étant donné ses dimensions.

Profitant de la période de fermeture annuelle du musée au mois de janvier 2017, une captation de la Tapisserie a été réalisée par la Fabrique de patrimoines en Normandie. L'opération, hautement compliquée, et qui aura mobilisé une cinquantaine de personnes pour accompagner en toute sécurité les déplacements de cette broderie vieille de près de 1000 ans, a permis de prendre diverses séries de 86 clichés photographiques couvrant l'ensemble de l'œuvre sous des modalités d'éclairage différentes: lumière du jour, réflexion infrarouge, réflexion et fluorescence ultraviolet...

Le nombre important de clichés s'explique par les fortes contraintes d'accès à l'œuvre qui, même sortie à l'intérieur du local technique hors de sa vitrine, reste suspendue à son rail sur un parcours trop rarement rectiligne et offrant trop peu de recul pour la mise en place de l'appareil servant à la captation dans des conditions garantissant la préservation de l'œuvre. Ces conditions complexes ont influé sur la qualité des clichés obtenus.

Toute une succession de traitements informatiques complexes lancés sur des serveurs de calculs puissants aura été nécessaire pour produire ce fond de manière quasi automatique avec une luminosité homogène, sans perte de données et contournant les problèmes de recalage dus au caractère «vivant» des matières textiles qui composent l'œuvre. Il faut maintenant proposer l'outillage nécessaire à sa visualisation ${ }^{23}$.

22. WiLson, 2005, p. 10: «Il faut cependant faire remarquer, à l'attention du visiteur moderne de Bayeux, qu'un peu de l'éclat des couleurs est perdu à cause de la nécessité où l'on est de tempérer l'intensité de la lumière, et de l'indispensable présence d'une vitre de sécurité devant la tapisserie elle-même.»

23. Voir aussi Zidane, El Moataz, Clouard, Daret et Berthelot, à paraître. 


\subsubsection{Mise en place de la présentation $d u$ «fond de référence»}

Notre «fond de référence» couvrant l'ensemble de la Tapisserie ainsi obtenu est une image qui possède des caractéristiques gargantuesques: le poids du fichier dépasse les $10 \mathrm{Go}$, avec une hauteur raisonnable de 5300 pixels pour une longueur importante de plus de 482000 pixels. Il est prévu de construire d'autres fonds similaires dans leurs caractéristiques représentant la Tapisserie via l'utilisation des autres modalités d'éclairage de la campagne de captation 2017 ou bien via des traitements d'images. La visualisation de toutes ces images hors normes est clairement impossible sans un dispositif approprié et des outils spécialisés comme il en existe dans le domaine des SIS et plus particulièrement dans les SIG pour la visualisation des fonds de cartes.

Un serveur d'image dédié au projet a donc été mis en place ${ }^{24}$. Celui-ci respecte les spécifications techniques de la norme $\operatorname{IIIF}^{25}$ dont l'objectif est de définir un cadre d'interopérabilité pour la diffusion d'images haute résolution sur le $\mathrm{Web}^{26}$. Chaque image complète de la Tapisserie aura été préalablement «tuilée» avant d'être intégrée au système afin de permettre une restitution plus fluide de l'œuvre via l'interface en ligne de l'outil de visualisation.

Cette visualisation, accessible par un accès personnel et sécurisé, est intégrée sur une page web, via l'implémentation d'une librairie JavaScript open source ${ }^{27}$, grâce à laquelle les utilisateurs bénéficient de fonctions classiques en termes d'affichage, d'interaction ou de navigation (déplacements, zooms, etc.). Mais l'objectif du projet ne s'arrête pas à la simple visualisation de l'œuvre, les capacités d'un SIS à superposer plusieurs couches d'informations vont être mises à contribution en particulier pour permettre l'indexation des données documentaires directement sur la Tapisserie (voir fig. 4).

\subsubsection{Référencement spatial des unités documentaires:} le système d'indexation

Plus qu'une représentation complète de l'œuvre, nous avons défini un espace de référencement qui sera utilisé pour indexer les éléments contenus dans la base de données documentaires sur la Tapisserie. À partir d'un repère de coordonnées cartésiennes dans ce système de projection appliqué à la Tapisserie, il est possible de définir géométriquement des zones d'intérêts, soit via des fonctions de dessin intégrées à la librairie Leaflet, soit par détection semi-automatique de formes en utilisant par exemple des techniques de segmentation d'image ou de recalage.

Quelle que soit la manière de définir ou découvrir les zones d'indexation, les formes géométriques utilisées pour la représentation de ces zones respectent les standards OpenGIS établis par l'Open Geospatial Consortium, à savoir:

\footnotetext{
24. Utilisation d'un serveur IIPImage (http://iipimage.sourceforge.net/) qui est notamment utilisé par le C2RMF (https://c2rmf.fr/visualisation-de-limagerie-scientifique-2d).

25. International Image Interoperability Framework (http://iiif.io).

26. Crane, 2017.

27. Leaflet: http://leafletjs.com.
} 
- point (primitive géométrique permettant de représenter une position spatiale);

- rectangle (appelé «enveloppe» dans la norme, qui est composé de 2 points: son coin de coordonnées maximales et son coin de coordonnées minimales);

- polygone (Primitive géométrique spécialisant une surface plane définie par une frontière extérieure et zéro ou plusieurs frontières intérieures, chaque frontière intérieure définissant un trou dans le polygone. Dans notre cas présent, nous nous arrêtons à la frontière extérieure).

Une fois la zone d'intérêt définie, il faut la caractériser en indiquant des renseignements tels qu'une catégorie, des mots-clés ou des commentaires, pour finalement y associer une ou plusieurs unités documentaires provenant de la base de données. Afin de rendre fiables et durables ces annotations ou ces indexations sur les zones d'intérêts et ainsi permettre leur exploitation numérique plus facile, le système d'identifiants pérennes $A R K^{28}$ sera utilisé pour chaque zone créée.

Toute cette démarche permettra à terme l'enrichissement de la connaissance de l'œuvre en apportant des renseignements complémentaires sur des portions de l'espace d'étude clairement identifiées. Ce travail d'indexation devra s'opérer sur le long terme, mais il sera possible, pour le faciliter, de s'appuyer sur les travaux existants sur la Tapisserie, et notamment de nombreux écrits qui proposent narrativement des indexations, parfois à l'aide de systèmes de repérage astucieux et précis.

\subsection{La problématique du système de localisation}

Depuis près de trois siècles d'écrits sur la Tapisserie, les chercheurs et écrivains utilisent différentes techniques de localisation afin de guider le lecteur et étayer leur discours, les obligeant souvent à proposer une reproduction associée, de plus ou moins bonne qualité et surtout limitée naturellement par les contraintes du support imprimé. Et malgré toutes ces initiatives, aucune technique de localisation n'a jamais fait autorité dans la communauté scientifique. Or les unités documentaires déjà existantes y font généralement référence. Il apparaît donc indispensable de pouvoir proposer à l'utilisateur une navigation ou un repérage selon ces modes de localisation connus.

\subsubsection{Le système "classique»: la numérotation narrative continue}

Ce principe de numérotation linéaire dans le sens horizontal est le plus couramment employé. Malheureusement, il manque de précisions dans l'ensemble des méthodes employées (par les limites de scènes, les inscriptions, l'iconographie, etc.) et varie d'un auteur à un autre. Aucun consensus n'ayant été trouvé, nous pensons qu'il n'est pas pertinent d'en implémenter un en particulier plutôt

28. Archival Resource Key: système pouvant garantir l'identification d'une ressource sur le long terme (http://www.bnf.fr/fr/professionnels/issn_isbn_autres_numeros/a.ark.html). 
qu'un autre. Pour autant, il est clairement possible d'identifier des éléments factuels incontestables qui pourront permettre aux utilisateurs d'améliorer leur expérience de navigation sur l'œuvre, sans suggérer de limites, mais en proposant d'utiliser certains éléments bien identifiés comme points d'ancrage. Pour cela nous avons d'ores et déjà identifié les éléments suivants:

- les numéros (de 1 à 58) apposés sur la bande numérotée, toile ancienne cousue au-dessus de la toile de base de la Tapisserie (fig. 5);

- les inscriptions latines.

Sans chercher à poser des limites de scènes sur la Tapisserie, le simple étiquetage de ces éléments factuels sur une barre de navigation horizontale permet au lecteur de se déplacer rapidement sur le support.

\subsubsection{Le système des lés}

Ce système de localisation, qui n'a été utilisé principalement que par des spécialistes du textile, présente l'avantage de prendre appui sur des limites objectives et matérielles: il s'agit des neuf panneaux de lin, appelés lés, identifiés par les restauratrices lors de la campagne de 1982-1983, composant l'ensemble de la toile brodée (fig. 6).

\subsubsection{Le système calque de la campagne 1982-1983}

Issu de la dernière campagne d'études en 1982-1983, ce système de repérage présente un intérêt primordial pour ce projet par les possibilités d'indexation automatique des unités documentaires créées à cette occasion et qui serviront de comparateur pour le futur constat sur l'état matériel de l'œuvre.

Afin de localiser précisément les informations de leurs différents diagnostics, les restauratrices chargées de l'étude ont quadrillé la Tapisserie sur des calques et dessiné manuellement les contours des motifs brodés (fig. 7). La localisation se base sur un système de coordonnées défini par ce quadrillage dont les carrés mesurent $10 \mathrm{~cm}$ de côté, ce qui rend le référencement des éléments précis, mais il demeure améliorable.

De plus, certaines imperfections de confection des calques, de l'avis même de leurs auteurs, limitent leur utilisation au repérage, sans pouvoir les considérer «comme une reproduction scrupuleuse de la Tapisserie ${ }^{29}$.

Malgré cela, le fait que les fiches documentaires et carnets de notes réalisés lors de cette campagne fassent référence à ce système de localisation nous a poussés à les exploiter. Un travail spécifique de traitements d'image a permis d'extraire les grilles de chaque calque pour ensuite les recaler sur le fond de référence. Chaque case du quadrillage devient alors une zone d'indexation spécifique, et les unités de la base documentaire qui vérifient cette référence y sont automatiquement indexées (fig. 8).

29. Bedat, Girault et Monier, 1982-1983, p. 9. 


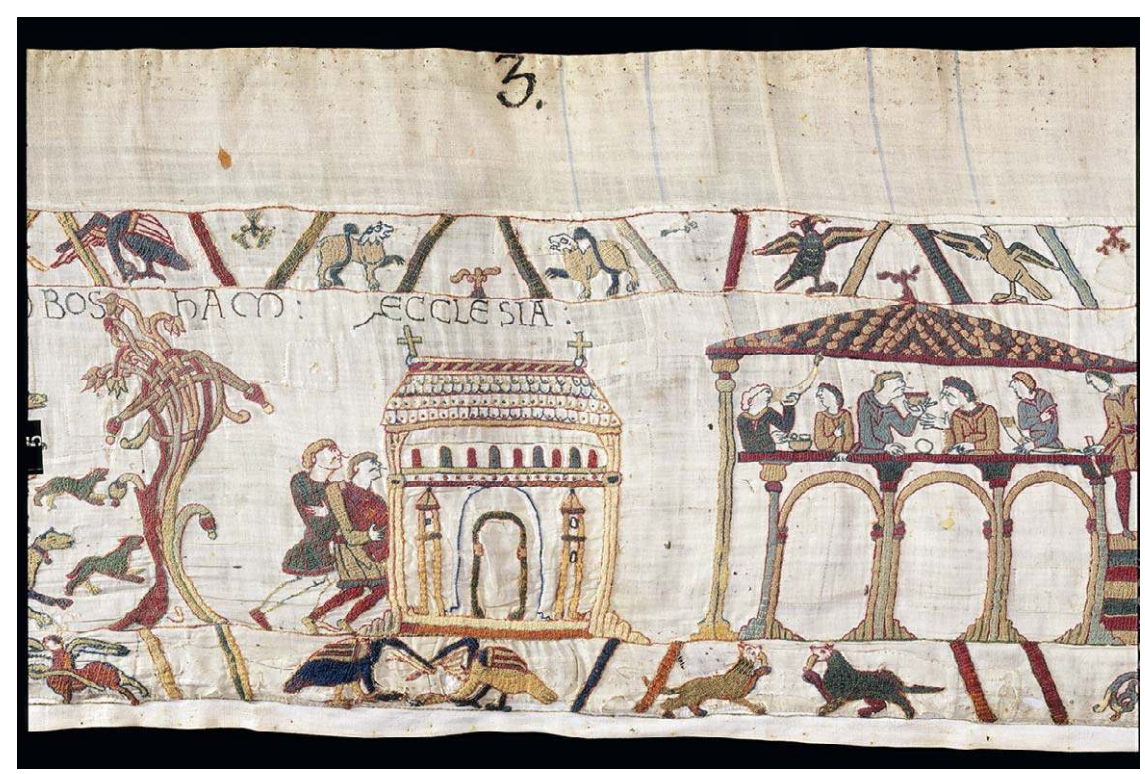

Fig. 5: Scène $\mathrm{n}^{\mathrm{o}}$ 3. Détail de la Tapisserie de Bayeux - $\mathrm{XI}^{\mathrm{e}}$ siècle. Avec autorisation spéciale de la Ville de Bayeux

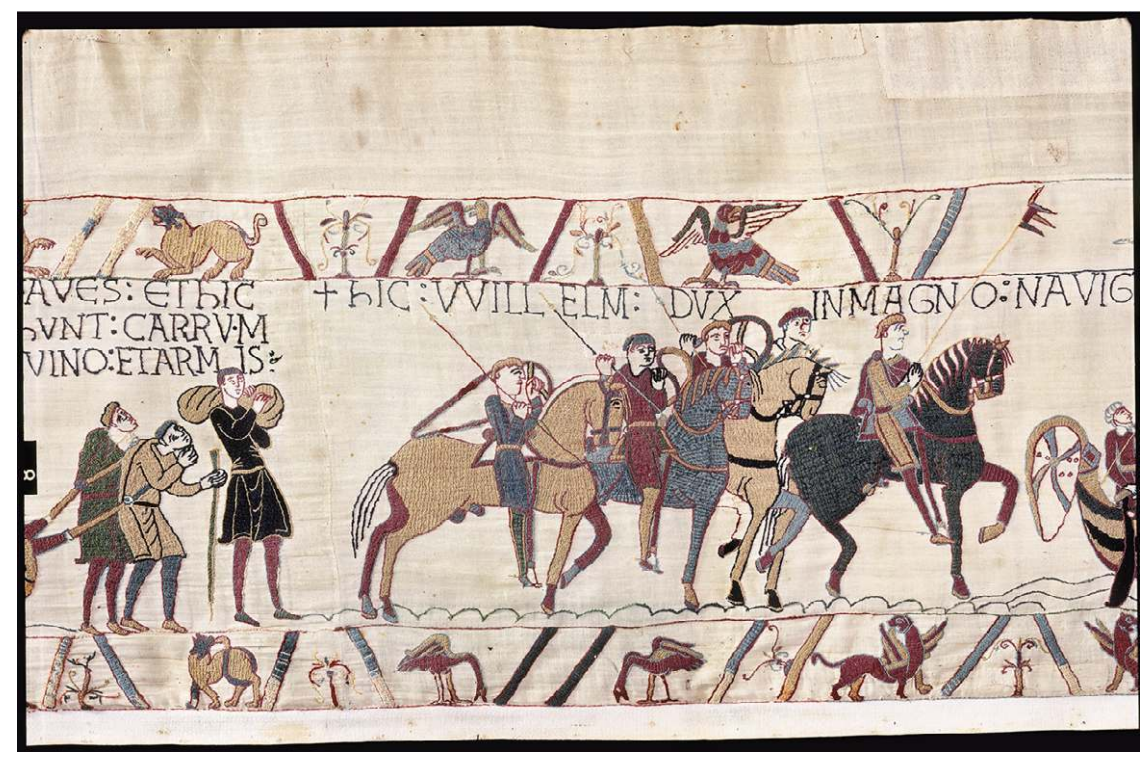

Fig. 6: Limite entre les lés $n^{0} 3$ et $n^{0} 4$.

Détail de la Tapisserie de Bayeux - $\mathrm{XI}^{\mathrm{e}}$ siècle.

Avec autorisation spéciale de la Ville de Bayeux 


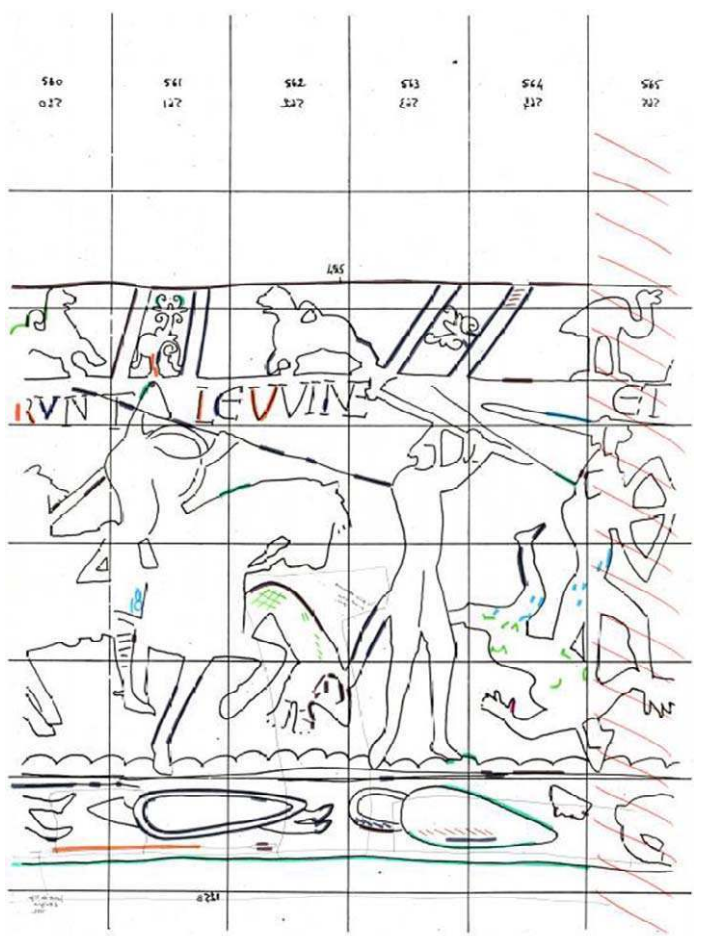

Fig. 7: Calque utilisé pour les relevés de la campagne de 1982-1983.

Calque Broderie $\mathbf{n}^{\circ}$ 125. Ministère de la Culture (France) -

Médiathèque de l'architecture et du patrimoine (Charenton-le-Pont), oo8o/168, I. Bédat, B. Girault, V. Monier

\subsubsection{Le référencement par l'iconographie (système «Lewis»)}

Système bien connu chez les Anglo-Saxons, il est le fruit du travail de l'historien britannique Michael Lewis qui a répertorié chaque élément iconographique à l'aide d'une syntaxe incrémentale basée sur les types de motifs: les personnages (sans préfixe), l'architecture (préfixe « $\mathrm{B}$ » pour buildings), les bateaux (préfixe « $\mathrm{S}$ » pour ships), les animaux (préfixe «A» pour animals), etc. (fig. 9).

Bien que, dans le cadre de notre projet, ce système soit incomplet - car ne prenant pas en compte la toile de support mais uniquement les éléments brodés - et imprécis (l'étiquetage d'un élément ne traduit pas de manière précise sa géométrie), il possède l'énorme avantage de pouvoir localiser des éléments indépendamment des variations de taille de l'œuvre selon sa tension.

Dans le cadre de notre méthode d'indexation, ce système Lewis peut parfaitement se confondre avec les zones d'intérêts créées, ce qui comblerait une partie de ses lacunes. Techniquement, cette correspondance pourra être réalisée via l'ajout d'une caractéristique spécialement dévolue au système Lewis pour chaque zone d'intérêt. 


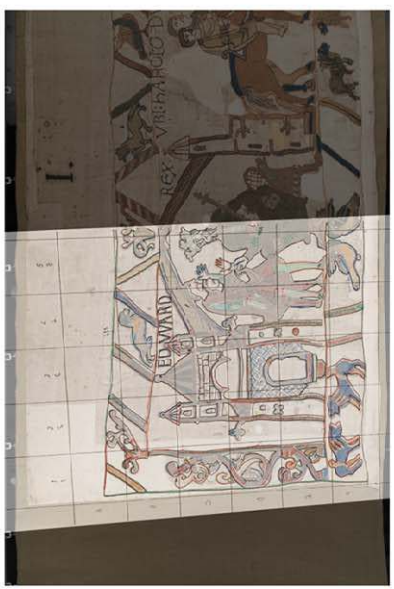

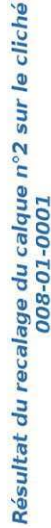

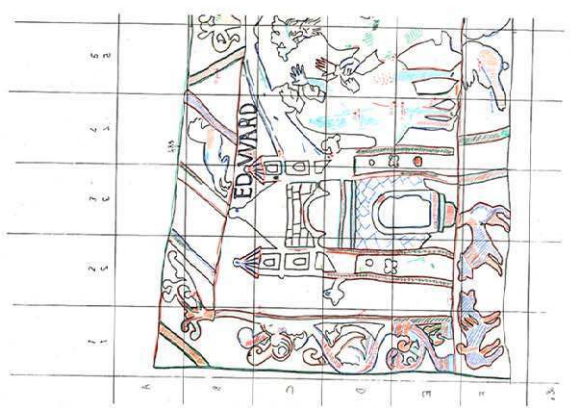

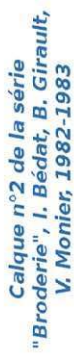

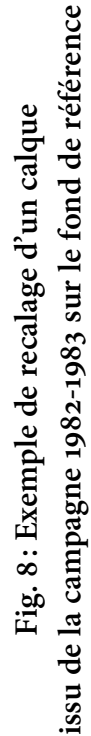
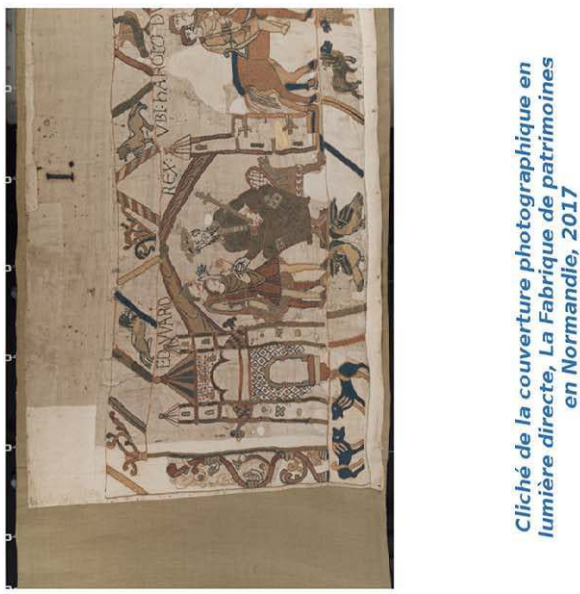

Tabularia «Études», «Les sources des mondes normands...», 2018, p. 1-27, 19 décembre 2018 


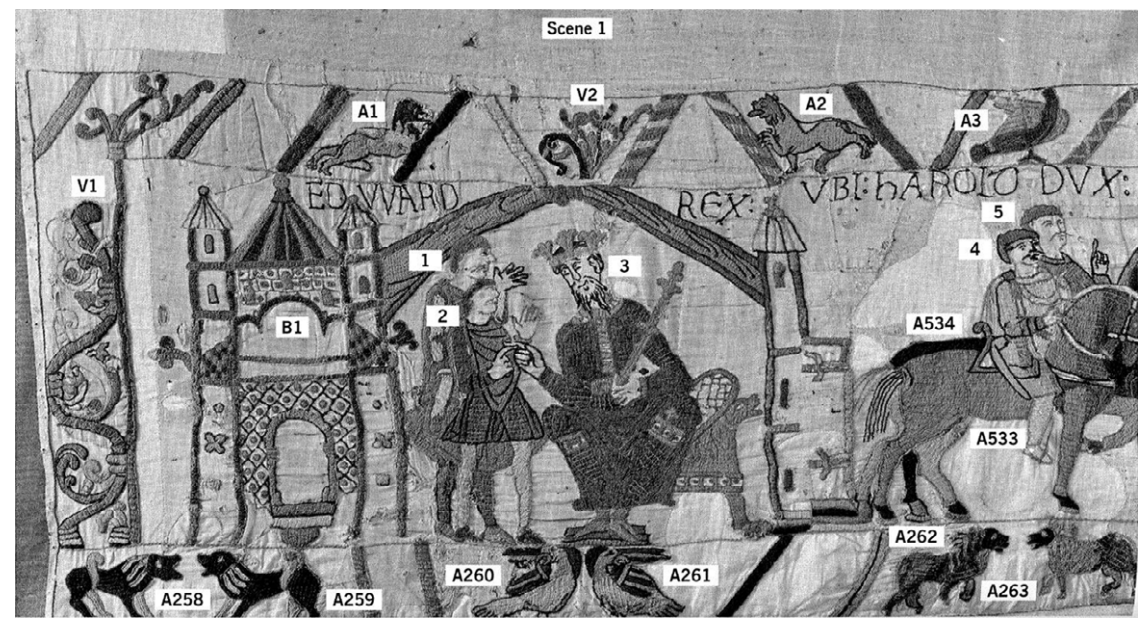

Fig. 9: Système de référence réalisé par Michael Lewis, planche $\mathrm{n}^{\mathrm{0}} 1$ dans LeWIS, 2005, p. 268

\subsubsection{Le calcul à partir d’images à échelles réduites (système «Freeman»)}

Le système mis en place par le chercheur Eric Freeman ${ }^{30}$ se veut très précis, car il utilise des mesures de distance sur un repère orthogonal à partir de deux référentiels bien connus dans le monde anglo-saxon: les gravures présentes dans les œuvres de Stenton de 1965 et celles de Wilson de 1985.

Ce système Freeman présente des incompatibilités fortes, car ces gravures tirées de Stenton et Wilson et utilisées comme référentiels présentent des tensions particulières. Ces mesures peuvent donc difficilement s'appliquer à d'autres clichés ou gravures. De même, ce système, très précis pour repérer un point, l'est beaucoup moins pour repérer un élément dans son ensemble si celui-ci est volumineux. Il faut de plus reprendre les mesures manuellement pour retrouver un élément sur la gravure de référence, ce qui est plutôt fastidieux.

Cependant, certaines bonnes idées utilisées dans ce système ont été améliorées et incorporées dans ce projet: utilisation d'un référentiel, avec un repère orthogonal et des mesures précises de distance. Il est en effet possible de se localiser d'une manière extrêmement rapide et facile sur notre visualiseur en ligne. L'apport du numérique et sa rapidité d'accès font de ce système Freeman une accroche intéressante dans le protocole final choisi pour notre projet.

\subsubsection{Le calcul à partir de l'œuvre elle-même (système «Hill»)}

Le système Hill est dérivé du système Freeman ${ }^{31}$. Contrairement à ce dernier, il se base sur une représentation complète de l'œuvre avec des mesures sur l'axe

\footnotetext{
30. FrEeman, 1991.

31. Hill, 2004.
} 
horizontal uniquement, la verticalité étant traitée par un découpage en trois zones: frise centrale, bordure inférieure et supérieure.

Bien que la localisation soit plus efficace que pour le système Freeman, elle demeure insuffisante sur le plan vertical, non résistante aux différences de tension de l'œuvre suivant la prise de vue étudiée, et ne conserve pas la bande numérotée, dont les numéros de scène sont largement exploités dans la littérature ou auprès du grand public.

Le principal atout de ce système de localisation réside dans l'utilisation de l'œuvre entière pour se localiser. Là encore, l'idée a été retenue dans le cadre de notre projet.

\subsubsection{Bilan sur la localisation}

Fort des atouts des systèmes de localisation existants, le système de visualisation et d'indexation mis en place dans ce projet permet donc de répondre à une des principales problématiques et de combler une lacune présente en général sur les systèmes de localisation existants: la possibilité de localiser de manière universelle, pérenne et précise des connaissances liées à la Tapisserie, qui a vocation à être accessible à l'ensemble de la communauté scientifique, au-delà de toutes considérations de langues ou de contraintes de reproduction de l'œuvre.

L'autre versant du projet réside en trois étapes qui se résument à trois types de publics différents: les conservateurs / restaurateurs qui doivent rédiger un nouveau constat sur l'état matériel de l'œuvre, les chercheurs qui par le biais de nouvelles modalités de représentation et d'accès aux informations liées à la Tapisserie vont pouvoir élargir leurs champs de recherche ou aborder les questions selon un angle nouveau, et enfin le grand public qui pourra bénéficier d'un outil de visualisation totalement inédit, plus dans l'air du temps, et à qui il sera proposé des médiations et des interactions nouvelles pour découvrir ou redécouvrir la Tapisserie.

Dans un premier temps, il faut permettre aux premiers utilisateurs concernés par ce projet - une équipe de conservateurs / restaurateurs - de pouvoir remplir sa mission: la réalisation d'un constat d'état sur l'état matériel de l'œuvre. Pour les accompagner dans leurs travaux, la visualisation est un premier atout: elle donne la possibilité d'offrir un premier regard sur la Tapisserie au-delà de la période de fermeture du musée où elle peut être accessible sans sa vitre de protection, ainsi que de bénéficier de la visualisation du revers de l'œuvre, face inaccessible en l'état puisqu'elle est recouverte d'une doublure posée au XVIII ${ }^{e}$ siècle $^{32}$. Elle permet surtout de centraliser, restituer et référencer spatialement les unités documentaires existantes, notamment de la précédente campagne, matériel nécessaire pour la rédaction du diagnostic.

32. Des photos du revers de la Tapisserie sont accessibles en ligne sur le site de la RMN (https://www. photo.rmn.fr/Package/2C6NUo5WWFF8), ainsi que sur un support tactile dans les espaces muséographiques du Musée.

Tabularia «Études», «Les sources des mondes normands...», 2018, p. 1-27, 19 décembre 2018 


\subsection{Restitution documentaire: le système d'extraction}

Une fois cette architecture de type SIS en place, nous bénéficions de différentes couches thématiques d'informations et d'un ensemble des données documentaires indexées sur notre système de projection. Nous pouvons alors proposer une interrogation à différentes échelles, en croisant les différentes caractéristiques des données qui composent chaque couche d'informations, en filtrant éventuellement les résultats via des thématiques d'indexation proposées (support, iconographie, broderie, etc.) mais aussi toute métadonnée mise à notre disposition.

Cette méthode d'extraction d'informations proposée à l'utilisateur bénéficie donc du travail de structuration des données du système documentaire et des capacités d'un SIS à naviguer sur l'œuvre, à afficher et à croiser des couches de données hétérogènes, en utilisant potentiellement des systèmes de localisation issus de la recherche scientifique.

Dans l'optique de faire de ce système et de son fond de référence un support d'étude universel, il est prévu de continuer à alimenter le système documentaire par des rapports sur l'état matériel de l'œuvre par des restaurateurs, par des études historiques ou scientifiques dans le cadre de travaux de recherche. Aussi, le résultat d'une extraction d'informations se veut-il déjà structuré, avec la possibilité d'annoter les données déjà présentes en proposant des modalités d'exploitation qui conservent les grands principes du système documentaire, à savoir respecter les standards internationaux de description des données et des normes en usage à des fins d'échanges, d'interopérabilité et de pérennisation des ressources ${ }^{33}$.

\section{Conclusion}

Le système documentaire doit donc répondre à la nécessité de mutualiser des documents d'envergures variables et pour autant conjointes: notices bibliographiques, accès aux contenus référencés par ces notices bibliographiques mais aussi contenus scientifiques sur l'état matériel de l'œuvre ou sur des aspects historiques à venir, produits depuis le SIDS lui-même et versés à la base de données documentaire, ou des documents provenant d'autres bases de données. À travers ces différents niveaux, l'enjeu est bien entendu de garantir la possibilité $\mathrm{du}$ dialogue scientifique dans des espaces, des temps et des langues de plus en plus larges en assurant la redocumentarisation de l'information propre à la Tapisserie.

L'utilisation des techniques des systèmes d'informations spatialisées apporte, quant à elle, une réponse probante aux problématiques de visualisation, de localisation et de mise en corrélation de l'information documentaire. Le fond de référence est aujourd'hui pratiquement finalisé. À court terme, plusieurs autres fonds seront générés puis recalés sur ce fond de référence, notamment à partir

33. Extractions au format XML TEI. 
des clichés du revers de la Tapisserie qui ont été captés pendant la campagne de restauration de l'œuvre en 1982-1983. Il sera donc possible de construire et croiser de nouvelles couches d'informations spécifiques, dont les données du système documentaire. L'utilisation de zones d'intérêts correctement caractérisées et annotées apporte également de nouvelles perspectives en termes d'analyse et d'étude grâce aux techniques employées dans un SIS via certains opérateurs géométriques: superposition, intersection, inclusion, union, distance, positionnement. Il sera ainsi possible de se poser des questions du type: combien y a-t-il de cavaliers armés sur la Tapisserie? ou encore: où sont représentés Harold et Guillaume ensemble sur la Tapisserie? La combinaison de ces techniques issues du domaine de l'information spatialisée avec celles utilisées dans la production, le traitement et l'analyse de données en information documentaire forme donc un système original, un support d'étude universel et novateur qui renforcera notre connaissance de cet ouvrage unique, élément majeur du patrimoine historique de l'Europe du Moyen Âge.

Il s'agira par exemple pour un restaurateur de pouvoir extraire facilement tous les clichés d'une zone de la Tapisserie surveillée régulièrement par le $\mathrm{LRMH}^{34}$. Plus généralement, ce système doit permettre à tous les experts restaurateurs ou chercheurs d'accéder facilement à d'importantes sources de données, en intégrant la numérisation de la Tapisserie en 2017 comme un référentiel pérenne d'étude.

\section{Bibliographie}

BéDAt, Isabelle, Girault, Béatrice, Monier, Véronique, Étude technique de la broderie de Bayeux. Rapport d'études menées du 2 novembre 1982 au 31 janvier 1983, rapport conservé à la Médiathèque de l'Architecture et du Patrimoine, cote 2009/013/074 (dactyl.), 1982-1983.

Bertrand, Simone, La Tapisserie de Bayeux et la manière de vivre au onzième siècle, La Pierre qui vire, Éditions Zodiaque, 1966.

Bouet, Pierre et Neveux, François, La Tapisserie de Bayeux. Révélations et mystères d'une broderie du Moyen Âge, Rennes, Éditions Ouest-France, 2013.

Brown, Shirley-Ann, The Bayeux Tapestry, Bayeux, Médiathèque municipale: ms. 1, A Sourcebook, Turnhout, Brepols, 2013.

Chefneux, Hélène, «Les fables dans la Tapisserie de Bayeux», Romania, vol. 6o, n 237, 1934, p. 1-35.

Crane, Tom, «An introduction to IIIF», Digirati, mars 2017. URL: https://resources. digirati.com/iiif/an-introduction-to-iiif/.

34. Le LRMH a sélectionné en 1985 une cinquantaine de zones fragiles ou tachées de l'œuvre, afin d'en faire le relevé photographique tous les dix ans (rapports LRMH de 1985, 1995, 2009 et 2014). 
Foys, Martin Kennedy, The Bayeux Tapestry Digital Edition, Woodbridge - Leicester, Boydell \& Brewer - SDE (CD), 2003. Disponible en ligne depuis 2013 au lien suivant: http://www.sd-editions.com/bayeux/zoom/\#/facsimile\%3DBayeux\%26panel\%3D2 (consulté le 15 décembre 2017).

Freeman, Eric F., "The Identity of Aelfgyva in the Bayeux Tapestry», Annales de Normandie, $41^{\mathrm{e}}$ année, $\mathrm{n}^{\circ}$ 2, 1991, p. 117-134.

Henderson, Anna C. et Owen-Crocker, Gale R. [éd.], Making Sense of the Bayeux Tapestry: Readings and Reworkings, Manchester, Manchester University Press, 2016.

Hill, David, «La Tapisserie de Bayeux: la reconstitution d'un texte», in La Tapisserie de Bayeux, L'art de broder l'Histoire, Pierre Bouet, Brian LÉvy et François Neveux (dir.), Actes du colloque de Cerisy-la-Salle, 1999, Caen, Presses universitaires de Caen, 2004, p. 383-402.

La Tapisserie de Bayeux, L'art de broder l'Histoire, Pierre Bouet, Brian LÉvy et François Neveux (dir.), Actes du colloque de Cerisy-la-Salle, 1999, Caen, Presses universitaires de Caen, 2004.

Lemagnen, Sylvette, La Tapisserie de Bayeux: une découverte pas à pas, Bayeux, Orep, 2015.

Lemagnen, Sylvette, Brown, Shirley-Ann et Owen-Crocker, Gale (dir.), L'invention de la Tapisserie de Bayeux: Naissance, composition et style d'un chef-d'œuvre médiéval, Actes du colloque international, Ville de Bayeux, 22-25 septembre 2016, Point de vues/Ville de Bayeux, à paraître en décembre 2018.

Lewis, Michael J., The Archaeological Authority of the Bayeux Tapestry, Oxford, British Archaeological Reports (British Series), 2005.

Nations-Unies, Division de statistique, Manuel des systèmes d'information géographique et de cartographie numérique, New York, Études méthodologiques. Série F, 79, 2004, p. 226.

Neveux, François, «Introduction», in La Tapisserie de Bayeux, L'art de broder l'Histoire, Pierre Bouet, Brian Lévy et François Neveux (dir.), Actes du colloque de Cerisyla-Salle, 1999, Caen, Presses universitaires de Caen, 2004, p. 11-14.

Pagès i Paretas, Montserrat, El Tapís de Bayeux, eina política? Anàlisi de les imatges i nova interpretació (trad. angl. Philip Banks), Barcelone, Publicacions de l'Abadia de Montserrat, 2015.

PArisse, Michel, La Tapisserie de Bayeux. Un documentaire du XI siècle, Paris, Éditions Denoël, 1983.

Pédauque, Roger T., Salaün Jean-Michel et Melot Michel (préface), Le document à la lumière du numérique, Caen, C\&F Éditions, 2006.

SALAÜN, Jean-Michel, «La redocumentarisation, un défi pour les sciences de l'information », Etudes de communication [En ligne], 30, 2007. URL: http://journals. openedition.org/edc/428.

Stenton, Frank, The Bayeux Tapestry ( $2^{\mathrm{e}}$ éd.), Londres, Phaidon press, 1965.

VIAL, Gabriel, Études techniques de la Broderie de Bayeux. Rapport du Centre International d'Étude des Textiles Anciens (CIETA), Lyon, novembre 1982 - janvier 1983, rapport conservé à la Médiathèque de l'Architecture et du Patrimoine, cote 2009/013/074. 
Wilson, David M., The Bayeux Tapestry, Londres, Thames and Hudson Ltd, 1985.

Wilson, David M., La Tapisserie de Bayeux, Paris, Flammarion, 2005.

Zidane, Katia, El Moataz, Abderrahim, Clouard, Régis, Daret, Arnaud et Berthelot, Clémentine, «La reconstruction du panorama de la Tapisserie de Bayeux comme fond de référence d'un Système d'Informations Géoréférencées ", In Situ, à paraître. 